\title{
UNA LEY DE EDUCACIÓN PARA DESTRUIR UN PAÍS
}

\author{
Antonio Jimeno ${ }^{1}$ \\ Presidente del Sindicato AMES, España
}

\section{Introducción}

El 29 de diciembre de 2020 en España se aprobó una nueva ley de educación denominada Ley Orgánica que Modifica la Ley Orgánica de Educación (LOMLOE)(5), también llamada "ley Celaá", en referencia a la actual ministra de educación, Isabel Celaá. Se aprobó en un tiempo record, pese a que la situación generada por la pandemia de la Covid-19, debido a que no se dejó intervenir a los expertos en educación del país, ni se consultó al Consejo de Estado de Educación, ni se intentó llegar al famoso Pacto de Estado por la Educación, que todo el pueblo español lleva pidiendo hace años, para acabar con el hecho de que cada vez que se produce un cambio de gobierno, el partido entrante hace una nueva ley de educación. La ley fue aprobada gracias a los votos de los partidos de izquierdas (PSOE, Unidas Podemos, Más País, Compromís y Nueva Canarias) y de dos partidos nacionalistas, es decir partidos que pretenden separar su región del resto de España (ERC y PNV). Votaron en contra los partidos de centro (Ciudadanos), de centro derecha (PP) y de derechas (VOX). El resto de partidos se abstuvo.

\section{Los antecedentes de la LOMLOE o ley Celaà}

En el año 1990 el PSOE aprobó una ley de educación llamada LOGSE(3), que empeoró significativamente nuestro sistema educativo porque aunque extendió la enseñanza obligatoria hasta los 16 años, lo cual estaba bien, ya que pasaba la enseñanza gratuita para todos de los 14 a los 16 años, que es la edad mínima para acceder al mundo laboral, lo hizo de la peor de las maneras posible, ya que estableció que todos los alumnos debían estar juntos en la misma aula y aprendiendo las mismas cosas hasta los 16 años, en lugar de permitir que antes pudieran escoger diferentes itinerarios en función de sus capacidades e intereses.

Como se sabía que habrían unos alumnos que aprobarían y otros que suspendían se estableció que en la Primaria (de 6 a 12 años) se permitiría repetir un curso, pero que después de

\footnotetext{
${ }^{1}$ Doctor em Ciencias Biológicas por La Universidad de Barcelona e Ingeniero Tecnico em Quimica Industrial por la Universidad Tecnica de Barcelona, catedrático de Instituto de Enseñanza. E-MAIL: ames@ames-fps.com.

Revista Tópicos Educacionais, Pernambuco, v. 27, n.01, p. 220-242, 2021. ISSN: 2448-0215.

https://periodicos.ufpe.br/revistas/topicoseducacionais/index 
esa repetición todos los alumnos, fuera cual fuera su nivel, pasarían a la Secundaria, a la que se llamó Educación Secundaria Obligatoria (12 a 16 años), vulgarmente conocida como "la ESO". En esta se permitió una repetición en $1^{\circ}$ o en $2^{\circ}$ de ESO y otra repetición en $3^{\circ}$ o en $4^{\circ}$ de ESO, siempre y cuando ya no hubieran repetido en la Primaria, ya que en total solo se podía repetir dos veces, es decir solo se podía estar en la enseñanza obligatoria hasta los 18 años (Artículo 6 de la LOGSE). Este diseño generó la llamada "promoción automática", es decir la promoción de curso del alumno que al haber suspendido ya varias veces sabía, desde el principio de curso, que estudiara o no estudiara, al año siguiente pasaría al curso siguiente. Nada más absurdo en un sistema educativo dirigido a adolescentes. El profesorado protestó pero no sirvió de nada porque tuvo que elegir entre aceptar la LOGSE o dejar la profesión y, lógicamente, la aceptó.

Los defensores del modelo LOGSE argumentan que repetir no sirve para nada y proponen que no se repita salvo en casos excepcionales, una solución que, por cierto, ahorraría mucho dinero al Estado. Se trata de una falacia porque claro que repetir en esas condiciones no sirve para nada, ya que el alumno sabe que al año siguiente promocionará sin esforzarse, lo que sí le estimularía a esforzarse es saber que si después de la repetición del $3^{\circ}$ o del $4^{\circ}$ de ESO no aprueba, ya no podría cursar el Bachillerato, ni la FP, salvo que superara una prueba especial de acceso.

Los ideólogos de esta ley lógicamente no fueron maestros de Primaria ni profesores de Secundaria, ni tampoco pedagogos como muchas veces se ha dicho, sino psicólogos como Alvaro Marchesi, catedrático de Psicología Evolutiva y de la Educación en la Universidad Complutense y su colega Cesar Coll, catedrático de la misma especialidad en la Universidad de Barcelona. Álvaro Marchesi ocupó altos cargos de Estado con los tres primeros ministros de educación del PSOE que impulsaron este sistema educativo que fueron José María Maravall (ministro de 1982 a 1988), Javier Solana (de 1988 a 1992) y Alfredo Pérez Rubalcaba (de 1992 a 1993).

Los ideólogos de este modelo nunca han dado clases ni en Primaria ni en Secundaria, ni han estudiado en un centro público o concertado de un barrio obrero, pero sin embargo sí se han considerado capacitados para hacer una ley de educación. Consideran que no es bueno separar a los alumnos en función de su rendimiento académico antes de los 16 años, porque si queremos una sociedad sin clases, sería un error separarlos ya en la escuela, por lo que se ha de promocionar de Revista Tópicos Educacionais, Pernambuco, v. 27, n.01, p. 220-242, 2021. ISSN: 2448-0215. https://periodicos.ufpe.br/revistas/topicoseducacionais/index DOI: $10.51359 / 2448-0215.2021 .250413$ 
curso a todos los alumnos, aunque algunos no estén preparados. No son conscientes de que un sistema educativo así desmotiva al alumno a esforzarse y, por lo tanto, lo está engañando porque no lo está preparando ni para continuar sus estudios ni para acceder a un trabajo ya que no habrá adquirido ni capacidad de esfuerzo ni hábitos de trabajo.

Aunque muchos políticos y periodistas siguen diciendo que después de la LOGSE en España han habido muchas otras leyes y en teoría es verdad, en la práctica los profesores siempre hemos tenido el mismo sistema educativo, el que estableció la LOGSE, ya que la siguiente ley, la LOCE(1) del PP, del año 2002, no se llegó a aplicar, y la LOE(2) del PSOE, del año 2006, cambió muy pocas cosas de la LOGSE, como era de esperar siendo del mismo partido, y la que vino luego, la LOMCE(4) del PP, del año 2013, cuya gran novedad era el establecimiento de unas evaluaciones externas con valor académico al final de la Primaria y al final de la ESO, justamente la medida indispensable para mejorar nuestro sistema educativo, fue congelada por el propio partido (PP) que la elaboró, y dichas evaluaciones nunca se llegaron a realizar. En resumen, en España llevamos treinta y un años, desde 1990 a 2021, con el mismo sistema educativo, el establecido por la LOGSE aprobada por el PSOE.

\section{Las pruebas PISA evidencian el fracaso de la LOGSE y de la LOE}

Para valorar el sistema educativo de un país no es objetivo atender a lo que diga su gobierno, sino comparar los conocimientos de sus alumnos con los de otros alumnos de su misma edad de otros países de un nivel económico parecido y a lo largo de varios años. Para ello van muy bien las pruebas internacionales como son las pruebas PISA, las TIMSS, las PIRLS, etc. En este artículo me referiré a las pruebas PISA porque se hace a alumnos de mayor edad, por lo que permiten comparar mejor los sistemas educativos. Son las pruebas internacionales de educación más importante del mundo.

Las pruebas PISA las diseña y las corrige la OCDE, mientras que su aplicación está en manos de cada país. Se hacen desde el año 2000, cada tres años, y su objetivo es valorar las competencias básicas de los jóvenes, es decir, aquellas habilidades que son necesarias para saber resolver los problemas sencillos de la vida ordinaria. Por esta razón, y porque tienen que ser las mismas en todos los países del mundo, sólo se valoran tres aspectos comunes: la comprensión Revista Tópicos Educacionais, Pernambuco, v. 27, n.01, p. 220-242, 2021. ISSN: 2448-0215. https://periodicos.ufpe.br/revistas/topicoseducacionais/index DOI: $10.51359 / 2448-0215.2021 .250413$ 
lectora, las matemáticas y las ciencias. Cada año se prioriza uno de esos tres aspectos. En las pruebas PISA 2018(9) se priorizó la comprensión lectora y, al igual que en 2015, no se han contestado en papel sino en ordenador, lo cual ha permitido conocer el tiempo empleado por cada alumno.

En la figura 1 se muestran los resultados de los 78 países participantes (PISA, 2018. Results)(10), ordenados de mayor a menor puntuación. Lo más sorprendente es que España no aparece en orden a su puntuación, sino al final de la figura 1, porque su calificación en "Comprensión lectora" fue tan baja que la OCDE decidió no publicarla. Debido a ello España, habiendo pagado lo mismo que otros países, no aparece en muchas de las comparaciones que se hacen con los otros 77 países respecto a múltiples variables. El origen de esta irregularidad radica, sobre todo, en la prueba de fluidez verbal, en la que se preguntaba la coherencia de 20 frases cortas, como por ejemplo “¿Los aviones están hechos de perros?”, que una proporción de nuestro alumnado contestó en solo 25 segundos y con una tasa de errores muy alta, cuando los alumnos de los demás países tardaron entre 50 segundos y 2 minutos. Este hecho es muy revelador del ambiente escolar que hay en algunos de nuestros centros, del poco interés que ponen bastantes de nuestros alumnos en hacer las cosas bien, porque no es que esos alumnos no supieran que los aviones no se hacen juntando perros, sino que pese a ser una prueba internacional, les daba igual contestar bien que mal, con tal de acabar cuanto antes.

Los resultados de España en las otras dos materias también fueron malos (ver la parte inferior de la figura 1): en Matemáticas solo se obtuvieron 481 puntos, cuando la media de la OCDE fue de 489 y en Ciencias solo se obtuvieron 483 puntos, cuando la media de la OCDE fue de 489 puntos. En conclusión, los alumnos españoles no llegaron a la media, es decir suspendieron, en las tres materias examinadas

Si nos comparamos con los demás países, sabiendo que 30 puntos en PISA equivale a los conocimientos adquiridos en un año de escolarización, resulta que en matemáticas, nuestros alumnos de 15 años tienen los conocimientos, por ejemplo, de los alumnos de 14 años de Estonia, de los de 13 años de Hong-Kong o de los de 12 años de las cuatro provincias chinas. En Ciencias sucede algo parecido. Todo ello es preocupante, ya que la comprensión lectora y los niveles de 
Revista Tópicos Educacionais

Revista do Programa de Pós-Graduação em Educação

da Universidade Federal de Pernambuco (UFPE)

matemáticas y ciencias aplicadas a la vida real, son esenciales para acceder al mundo laboral o para continuar estudiando con posibilidades de éxito.

\section{Resultados PISA 2018}

\section{Panorama del rendimiento en lectura, matemáticas y ciencias}

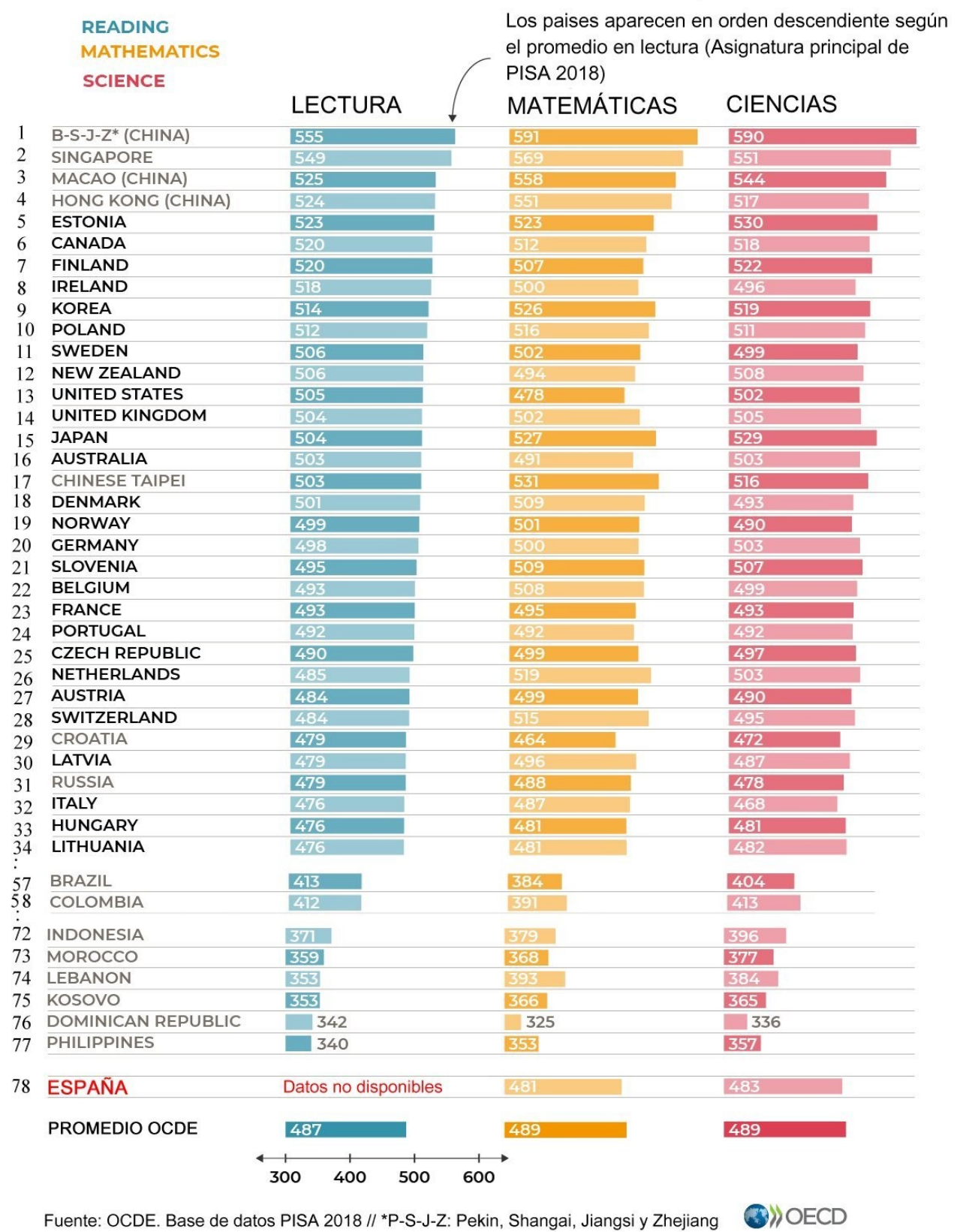

Figura 1. Clasificación de los países en las pruebas PISA 2018. Results(10) ordenados por su calificación en "Lectura".

Lo más importante para valorar el sistema educativo de un país no es fijarse en la posición que ha quedado sino observar su tendencia desde las anteriores pruebas, ya que ello

Revista Tópicos Educacionais, Pernambuco, v. 27, n.01, p. 220-242, 2021. ISSN: 2448-0215.

https://periodicos.ufpe.br/revistas/topicoseducacionais/index DOI: 10.51359/2448-0215.2021.250413 
Revista Tópicos Educacionais

Revista do Programa de Pós-Graduação em Educação da Universidade Federal de Pernambuco (UFPE)

puede permitir detectar qué cambios en las estrategias educativas están dando mejores resultados.

En la figura 2, se muestra cómo han ido variando los resultados de los países que por su proximidad o por sus buenos resultados, nos han parecido los referentes más significativos para España.

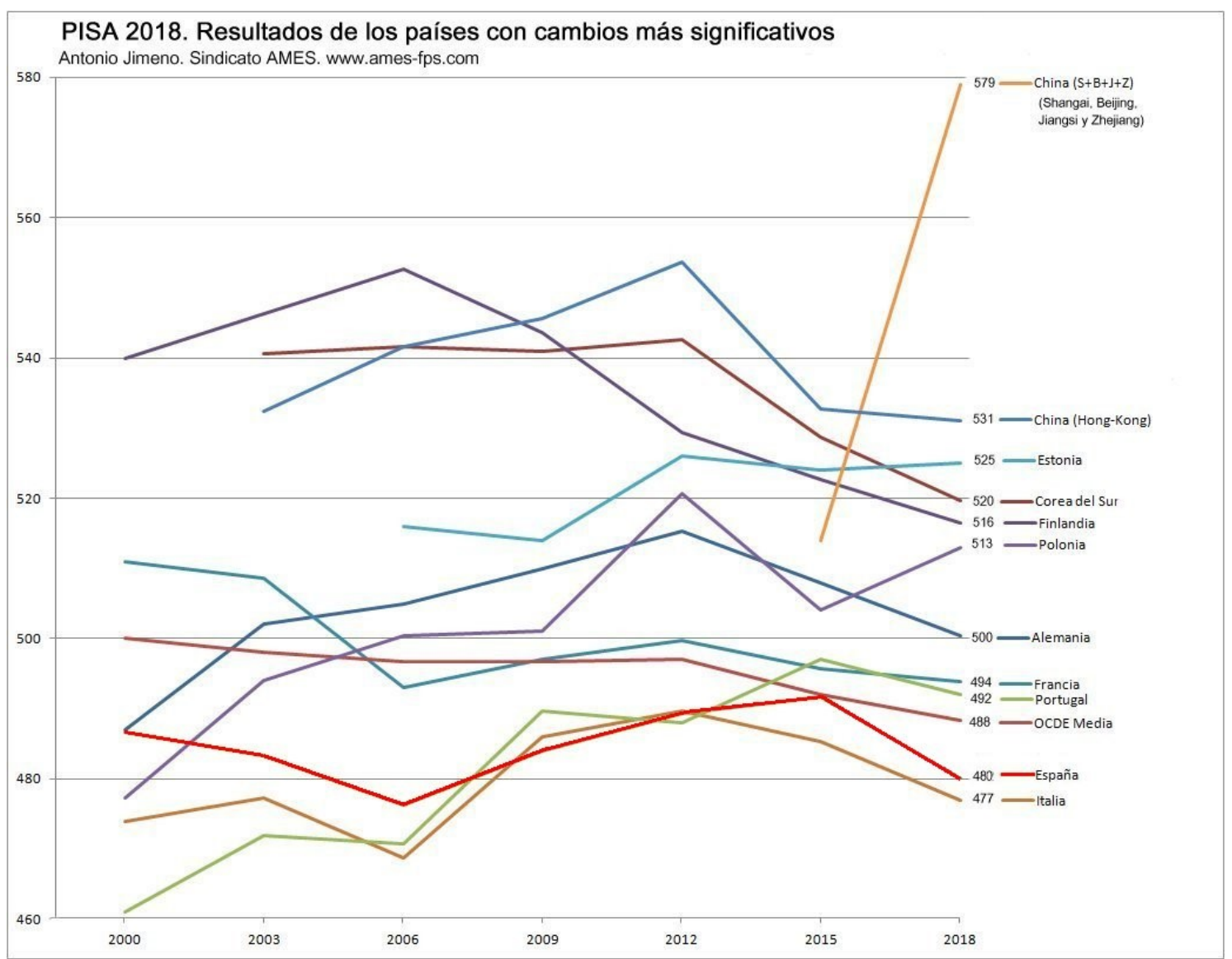

Figura 2. Resultados en las pruebas PISA de los países más significativos desde el año 2000 al 2018. Autor: Antonio Jimeno 2021. Fuente de datos OCDE. Informes PISA

En la figura 1 se puede observar que durante los 18 años que llevamos de pruebas PISA, los alumnos de España siempre han estado por debajo de la media de la OCDE, es decir siempre han suspendido las pruebas PISA, exceptuando las del año 2015 en las que simplemente llegaron a la media. El otro aspecto a resaltar es que el último descenso experimentado ha sido el más acusado. La conclusión es evidente nuestro sistema educativo, el denominado LOGSE/LOE, no funciona.

El país que más ha mejorado desde PISA 2015 ha sido el conjunto de las siguientes cuatro regiones de China: Shanghai, Beijing, Jiangsu y Zuangdong $(\mathrm{S}+\mathrm{B}+\mathrm{J}+\mathrm{Z})$. Suman 180 
millones de personas y han experimentado una espectacular mejora de 64 puntos, alcanzando una media de 579 puntos, desbancando así al pequeño Singapur, de 5,6 millones de habitantes, que fue el primer país en 2015. Conviene destacar que en China, como en el resto de países orientales, se realizan evaluaciones de Estado al final de las etapas educativas.

Entre los países con más de 420 puntos, el siguiente que más ha mejorado ha sido Polonia, que con una mejora de 9 puntos ha alcanzado los 513 puntos, ocupando así la tercera posición en Europa, después de la pequeña Estonia (525) y de Finlandia (516). El secreto del éxito de Polonia, que supera a España en 30 puntos pese a tener un PIB que es la mitad del español, se debe a haber aumentado la autonomía de los centros en cuanto a la forma de organizarse, instaurar evaluaciones nacionales estandarizadas ("reválidas"), establecer incentivos para los docentes y fomentar el aprendizaje basado en la experimentación, la investigación y la resolución de problemas.

En cambio, Portugal, que fue el país revelación en las PISA 2015, al experimentar una mejora de 9 puntos respecto a 2012, en 2018 ha sufrido un descenso de 5 puntos quedando con 492. Este brusco cambio de tendencia en Portugal coincide con el hecho de que en 2012, el ministro Nuno Crato estableció unas evaluaciones de Estado al final de la enseñanza Secundaria y las mantuvo pese a las protestas de $\operatorname{los} \operatorname{alumnos}(8)$ y de los sindicatos de profesores, mientras que en 2015, pese al éxito aportado por dichas pruebas, el ministro siguiente, Tiago Brandao, las retiró sin explicar los motivos. Otro país a destacar es Alemania, que a partir de los malos resultados en las PISA 2000, que obtuvo 487 puntos, los mismos que España, reaccionó y ahora se mantiene muy por encima del nivel de la OCDE. Tiene una muy buena FP, que inicia ya a los 13 años, lo cual le ayuda a tener unos valores de equidad altos, es decir, con pocos alumnos con rendimientos académicos bajos.

La principal conclusión que se puede deducir de comparar las tendencias, es que las evaluaciones externas por parte del Estado, son un elemento esencial para mejorar un sistema educativo. Como decía el anterior director del Instituto de Evaluación Educativa de Portugal, João Maroc: "Los exámenes consiguen una doble objetivo: comprometen a los profesores a cumplir el programa y comprometen al estudiante, porque sabe que va a tener un examen al final". 


\section{Los defensores de la LOGSE y de la LOE no quieren aceptar su fracaso}

Los sindicatos de clase han argumentado que este fracaso se debe a la falta de inversión en educación y que todo se arreglaría aumentando el número de profesores y disminuyendo el número de horas de clases a impartir. Es evidente que esto ayudaría, pero deberían reconocer que solo con eso no se arreglaría el problema. Por ejemplo, durante la última década en muchos países de la OCDE se ha aumentado hasta un 15\% las inversiones en educación y, sin embargo, apenas ha habido mejoras. Por otro lado tenemos el ejemplo de Polonia que con una inversión mucho menor que en España, nos ha superado en 30 puntos. En la figura 3 se puede observar que simplemente invertir más en educación, no quiere decir mejorar los resultados, hay que mejorar el sistema. Por ejemplo, Irlanda, siendo el penúltimo país en porcentaje de su PIB invertido en educación, ocupa el cuarto lugar de los 29 países comparados en cuanto a resultados en las PISA 2018, y Suecia, Dinamarca y Bélgica pese a ser los que más invierten, más del 6\%, no están entre los primeros.

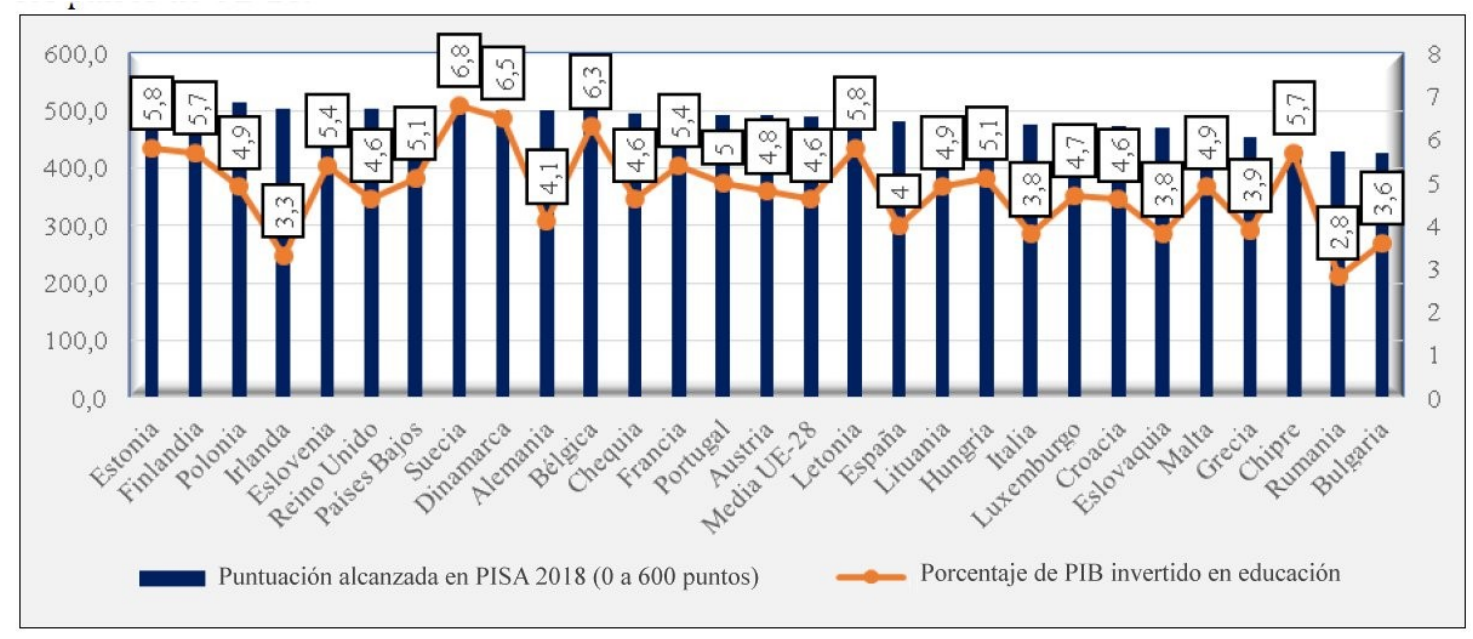

Figura 3. Puntuación media de PISA 2018 y porcentaje del PIB (2017) dedicado a educación en los países de la UE-28. Autora: Sánchez Lissen, E. 2020(13). Fuente: Ministerio de Educación y Formación Profesional, 2019.

Algunos famosos catedráticos de psicología evolutiva de la educación atribuyen el fracaso a que el profesorado no está bien preparado. Ellos piden que se aumente el número de años de formación y, lógicamente, ofrecen sus cursos y másteres en sus Universidades para solucionar el problema. Es evidente que cuanta más formación reciban tanto mejor, pero lo que les hace falta no es más teoría psicológica educativa, sino un mayor conocimiento de lo que han de enseñar y 
más años de práctica docente supervisada. Por otro lado, ellos omiten que tampoco el profesorado con muchos años de experiencia y con reconocido prestigio, consigue que el alumnado trabaje y aprenda al ritmo que lo hacía antes. Es absurdo e intolerable considerar que todos los profesionales de la docencia de un país, algunos de los cuales llevan toda su vida enseñando, no saben hacer su trabajo, y no pensar que el problema no es el profesorado sino el nuevo sistema que se ha impuesto.

Algunos docentes opinan que el fracaso se debe a que la enseñanza tradicional no funciona y que todo se arreglaría si se hiciera de forma diferente. Ellos proponen, por ejemplo, no impartir asignaturas ni seguir libros de texto, sino aprenderlo todo exclusivamente mediante la elaboración de proyectos por parte de equipos de 4 o 5 alumnos, no memorizar nada, estudiar algunas materias, especialmente las ciencias, en inglés, aunque se aprenda menos de esa materia, cosa que podría explicar el espectacular descenso en ciencias en las PISA 2018, el que hayan varios profesores en el aula o codocencia, agrupar varias materias y estudiarlas conjuntamente, lo que se denomina estudiar por ámbitos, etc. Es verdad que en la enseñanza, como en los demás sectores, una manera de progresar es probando nuevas estrategias, pero lo que no se puede pretender es que la Administración educativa no evalúe los resultados, es decir que no valore si con el nuevo sistema los alumnos están aprendiendo más o menos. En conclusión, sí a las innovaciones, pero de forma experimental, solo en algunos centros, durante un período corto de tiempo y siempre acompañados de pruebas externas para valorar los resultados.

Los partidos españoles de izquierdas, como PSOE y Unidas Podemos, que defienden el modelo LOGSE/LOE, han atribuido el fracaso en las PISA 2018 a la ley del PP, la LOMCE, sin decir que lo más importante de dicha ley, que son las evaluaciones externas con valor académico al final de la ESO y los dos itinerarios en $4^{\circ}$ de ESO, no se han llegado a aplicar nunca, porque las congeló el propio PP, ante las presiones de los demás partidos. Así pues, la LOMCE no ha sido la causa del problema, sino, a tenor de los buenos resultados de los países que tienen evaluaciones de Estado, como son los orientales, Polonia y Portugal en 2015, ha sido su no aplicación lo que ha provocado el fracaso en las PISA 2018.

Estos partidos están en contra de las reválidas al final de la ESO, porque no todos los alumnos las superarían y eso contradice su objetivo de que todos los ciudadanos tengan la ESO Revista Tópicos Educacionais, Pernambuco, v. 27, n.01, p. 220-242, 2021. ISSN: 2448-0215. https://periodicos.ufpe.br/revistas/topicoseducacionais/index DOI: 10.51359/2448-0215.2021.250413 


\section{Revista Tópicos Educacionais}

aprobada y, así, todos puedan acceder a las siguientes etapas educativas. Por ello defienden que todos los alumnos deben estar en las mismas aulas y aprendiendo las mismas cosas hasta los 16 años y, en el caso de repetir curso, hasta los 18 años. Esto es un grave error, porque a partir de los 14 o 15 años las diferencias de capacidad y de intereses pueden ser tan grandes, que mantenerlos juntos es perjudicarlos a todos. Es mucho mejor establecer varios itinerarios a partir de los 15 años, para que cada alumno elija el que más le interese, que generalmente es aquel en el que puede sacar mejores calificaciones.

Los partidos españoles nacionalistas, es decir los que pretenden separarse del resto de España, como son ERC, PNV y Bildu, también están en contra de las evaluaciones finales de ESO, pero por otro motivo, es porque al estar diseñadas, aplicadas y corregidas por el Ministerio, en ellas se detectaría el bajo nivel de castellano o español y el gran desconocimiento de la geografía y de la historia de España que tienen los alumnos de sus Comunidades Autónomas, por lo que obtendrían calificaciones muy bajas en estas dos materias y podrían no superar dichas evaluaciones.

\section{Los diez principales errores de la LOMLOE}

La LOMLOE fue aprobada el 29 de diciembre de 2020 (BOE del 30/12/2020) y tres meses después, el 26 de marzo de 2021, la ministra Isabel Celaá avanzó las líneas generales de los futuros nuevos currículos mediante los documentos "Propuesta de estructura curricular para la elaboración de las enseñanzas mínimas"(11) y "Perfil de salida del alumnado al término de la educación básica”(12), los cuales, según el calendario de implantación de la LOMLOE(6), se aplicarán a partir del curso 2022/2023. A continuación se exponen los principales diez errores pedagógicos de la LOMLOE y de los dos documentos bases sobre los currículos:

1) El primero de los grandes errores educativos de la LOMLOE es la retirada de las evaluaciones externas con valor académico, las llamadas reválidas, al final de la ESO. Estas pruebas fueron establecidas en el Artículo 29 de la ley anterior, la LOMCE, mientras que en el Artículo 29 de la LOMLOE ya no aparecen. El motivo por el que ello es un gran error es porque al no existir ningún control externo de lo que realmente saben los alumnos por parte del Ministerio de Educación, los centros, aunque pretendan mantener los niveles de exigencia, se ven obligados a Revista Tópicos Educacionais, Pernambuco, v. 27, n.01, p. 220-242, 2021. ISSN: 2448-0215. https://periodicos.ufpe.br/revistas/topicoseducacionais/index DOI: 10.51359/2448-0215.2021.250413 
aprobar a bastantes alumnos que, en realidad, no han llegado a los mínimos establecidos ni se han esforzado en ello, porque si no los aprobaran, al año siguiente estos alumnos se matricularían en otros centros más permisivos (cada vez más abundantes porque no hay control) y, pasados unos pocos años, el colegio debería cerrar por falta de alumnos. Es lo que se llama el síndrome del "alumno cliente".

Otro problema derivado de la ausencia de estas reválidas es que muchos de los alumnos que se esfuerzan y estudian, al ver que también pasan de curso sus compañeros que no lo hacen, acaban esforzándose menos y, por lo tanto, aprendiendo menos.

Por otro lado, en una situación como la de la España actual, en la que se multiplican los casos de adoctrinamiento político partidista en las aulas, debido a que algunos gobiernos autonómicos las están utilizando como canteras de futuros votantes a favor del secesionismo del resto de España; y en un momento en el que en varias Comunidades Autónomas (Cataluña, Baleares, Comunidad Valenciana y País Vasco), se ha impuesto una reducción o una prohibición del uso del castellano o español como lengua de comunicación entre profesores y alumnos, sólo mediante las reválidas al final de la ESO, podría el Ministerio de Educación detectar si se está adoctrinando políticamente a los alumnos en el secesionismo, si la Historia de España y la estructura del Estado que se les está enseñando es la real, es decir la contenida en la Constitución española, si el nivel de castellano o español alcanzado es el establecido, y si las calificaciones que los centros dan a las familias realmente reflejan los conocimientos alcanzados por los alumnos o es más elevada para así contentar a los padres y a los gobiernos autonómicos.

En conclusión, es un grave error que la ley Celaá haya eliminado estas pruebas porque, desde una perspectiva pedagógica, el hecho de tener que superar una prueba externa sería el gran estímulo que actualmente necesitan muchos alumnos de ESO para esforzarse más y así adquirir hábitos de trabajo. Sin estas reválidas se está poniendo en peligro el futuro del país, ya que si los jóvenes tienen pocos conocimientos y poca capacidad de esfuerzo, difícilmente España va a continuar siendo un país competitivo.

2) El segundo de los grandes errores de la ley Celaá es impedir que en $4^{\circ}$ de ESO hayan dos itinerarios, como establecía la ley anterior, la LOMCE, en su Artículo 25, uno itinerario dirigido hacia la FP y otro dirigido hacia el Bachillerato, dos itinerarios en los que se hubiera Revista Tópicos Educacionais, Pernambuco, v. 27, n.01, p. 220-242, 2021. ISSN: 2448-0215. https://periodicos.ufpe.br/revistas/topicoseducacionais/index DOI: $10.51359 / 2448-0215.2021 .250413$ 


\section{Revista Tópicos Educacionais}

podido preparar a los alumnos para dos tipos diferentes de evaluaciones finales de ESO. Esta estructura es indispensable porque a cada alumno solo se le debe pedir aquello que puede aprender y que le conviene para sus estudios posteriores. Mantener juntos en la misma aula, aprendiendo las mismas cosas, a todos los alumnos hasta los 16 años, o hasta los 18 años en el caso de los alumnos que repiten curso, es perjudicar tanto a los que luego quieren cursar un Bachillerato como a los que quieren hacer una FP. A los primeros porque se avanza poco en las materias teóricas y a los segundo porque no se les está enseñando las materias prácticas que necesitan aprender.

3) El tercero de los graves errores de la señora Celaá es haber rebajado los niveles de exigencia, estableciendo que las juntas de evaluación de cada centro puedan dar el título de la ESO aunque los alumnos tengan muchas asignaturas suspendidas (Artículo 28) y el título de Bachillerato aunque los alumnos tengan una asignatura suspendida (Artículo 37).

En el artículo 28 de la ley anterior, la LOMCE, la exigencia en la ESO era mayor, ya que se establecía que "repetirán curso cuando tengan evaluación negativa en tres o más materias, o en dos materias que sean Lengua Castellana y Literatura y Matemáticas de forma simultánea", criterios de exigencia que han desaparecido en la LOMLOE. Por otro lado, en el artículo 36 bis de la ley anterior, la LOMCE, la exigencia también era mayor en el Bachillerato ya que para tener aprobado el Bachillerato era indispensable tener aprobadas todas las asignaturas. Establecer que se puede aprobar una etapa pese a tener asignaturas suspendidas es un enorme disparate, porque lo primero que van a hacer bastantes alumnos es abandonar aquellas asignaturas que les cuestan más desde el primer día de clase, sabiendo que la ley les permite promocionar de curso aunque no las aprueben. Esto va a generar muchos problemas de orden en clase, en aquellas materias en las que por su dificultad haya muchos alumnos que las han abandonado. Puede que estos alumnos no vayan a clase o que, si se les obliga a ir al aula, acaben siendo alumnos conflictivos. Debido a este error aumentará el número de alumnos con el título de ESO y con el título de Bachillerato que tendrán varias materias suspendidas, a veces las más importantes para poder aprovechar los estudios posteriores. Esto es lo contrario a fomentar la capacidad de esfuerzo y el hábito de trabajo y de nuestros jóvenes.

4) El cuarto de los grandes errores de la LOMLOE es delegar totalmente en las Comunidades Autónomas el uso del castellano o español como lengua vehicular de enseñanza. En Revista Tópicos Educacionais, Pernambuco, v. 27, n.01, p. 220-242, 2021. ISSN: 2448-0215. https://periodicos.ufpe.br/revistas/topicoseducacionais/index DOI: 10.51359/2448-0215.2021.250413 
el punto 1 de la disposición adicional trigésima octava de la ley anterior, la LOMCE, se decía: "Las Administraciones educativas garantizarán el derecho de los alumnos y alumnas a recibir las enseñanzas en castellano, lengua oficial del Estado, y en las demás lenguas cooficiales en sus respectivos territorios. El castellano es lengua vehicular de la enseñanza en todo el Estado y las lenguas cooficiales lo son también en las respectivas Comunidades Autónomas, de acuerdo con sus Estatutos y normativa aplicable.”. En cambio, en ese mismo punto de la LOMLOE se ha suprimido la frase "El castellano es lengua vehicular de la enseñanza en todo el Estado". Al haberlo hecho, si alguien denuncia que en un centro educativo o en una Comunidad Autónoma se ha excluido totalmente el español como lengua de comunicación, como pasa en Cataluña, ya no podrá fundamentar su denuncia en la ley de educación, la LOMLOE, sino solo en el artículo 3 de la Constitución Española y en las sentencias de los tribunales españoles que establecen que como mínimo se ha de impartir un $25 \%$ de las clases en español. Lamentablemente el gobierno de Cataluña recurre esas sentencias y si pierde, solo las cumple en las aulas donde está el alumno denunciante y durante su período de escolarización, en vez de aplicarlas en todas las aulas del centro, en todos los centros de Cataluña y para siempre.

Aunque en el punto 3 se diga que "Las Administraciones educativas aplicarán los instrumentos de control ..... de modo que se garantice que todo el alumnado alcance la competencia en comunicación lingüística, en lengua castellana y en su caso en las lenguas cooficiales, en el grado requerido.”, dado que en varias de las Comunidades Autónomas ya no se utiliza el castellano o español como lengua de comunicación entre profesores y alumnos, que el término “Administraciones" se refiere a las Consejerías de educación autonómicas, y que no existe una evaluación externa por parte del Ministerio de Educación que permita constatar si los alumnos realmente han alcanzado el nivel de español establecido, estas frases no aseguran ese derecho, sino todo lo contrario, solo sirven a los autores de la LOMLOE para defender la constitucionalidad de la ley ante una denuncia en el Tribunal Constitucional español, ya que pueden argumentar que la propia ley ya asegura ese derecho.

5) El quinto error de la LOMLOE es permitir a los centros de las Comunidades Autónomas que lo permitan, dejar de impartir las asignaturas establecidas en la ley y, en su lugar, hacer las actividades que ellos decidan y calificar a sus alumnos no en función de lo que saben sobre los contenidos de cada asignatura, sino en relación a los aspectos que ellos consideren Revista Tópicos Educacionais, Pernambuco, v. 27, n.01, p. 220-242, 2021. ISSN: 2448-0215. https://periodicos.ufpe.br/revistas/topicoseducacionais/index DOI: $10.51359 / 2448-0215.2021 .250413$ 
oportunos. Esto se establece en el Artículo 120. 4, donde dice: "Los centros, en el ejercicio de su autonomía, pueden adoptar experimentaciones, innovaciones pedagógicas, programas educativos, planes de trabajo, formas de organización, normas de convivencia o ampliación del calendario escolar o del horario lectivo de ámbitos, áreas o materias, en los términos que establezcan las Administraciones educativas ...". Al no haber ninguna prueba externa que permita al Ministerio o a la Consejería de Educación evitar que obtengan el título de la ESO los alumnos que no han llegado a los mínimos establecidos, se abre el campo al descontrol absoluto de lo que se hace en los centros y al fraude educativo al alumno, a su familia y a toda la sociedad en general.

6) El sexto error de la LOMLOE es dejar en manos de las Comunidades Autónomas la enseñanza o no de las principales religiones. Mientras en la ley anterior, la LOMCE, la enseñanza estaba claramente establecida en el artículo 18.3 para la Primaria, en el Artículo 24.4 para la ESO y en el Artículo 34 para el Bachillerato, en la LOMLOE simplemente se ha modificado el apartado 3 de la disposición adicional segunda, que ha quedado redactado en los siguientes términos: “3. En el marco de la regulación de las enseñanzas de Educación Primaria y Educación Secundaria Obligatoria, se podrá establecer la enseñanza no confesional de cultura de las religiones.”. Decir que una materia como es el conocimiento de las principales religiones se podrá o no enseñar es un grave error. Es imposible entender la historia de los pueblos, sus tradiciones y su arte sin unos mínimos conocimientos de las religiones. Lo que se debería haber hecho es establecer que todos los alumnos, independientemente de si son creyentes o no, deben conocer las principales religiones del mundo. El conocimiento de las religiones debe ser una materia obligatoria, evaluable y con el mismo peso en la nota final que las demás asignaturas.

7) El séptimo error de la LOMLOE es que no existiendo una evaluación externa al final de la enseñanza obligatoria, se establezca que para las “enseñanzas mínimas”, es decir las que no pueden faltar, el Ministerio de Educación solo se reserve el 50\% de las horas en aquellas Comunidades Autónomas que tengan una segunda lengua cooficial, y el 60\% en las que no la tengan (Artículo 6.4). Esto todavía es más grave ya que ese escaso porcentaje está supeditado a lo que decida hacer cada centro, ya que los centros dispondrán de autonomía pedagógica, de organización y de gestión (Artículo 120.1). Debido a ello a las enseñanzas mínimas habría que haberle destinado mucho más tiempo en el horario escolar. La consecuencia de este error va a ser que en España haya 17 sistemas educativos diferentes, uno por cada Comunidad Autónoma. Revista Tópicos Educacionais, Pernambuco, v. 27, n.01, p. 220-242, 2021. ISSN: 2448-0215. https://periodicos.ufpe.br/revistas/topicoseducacionais/index DOI: $10.51359 / 2448-0215.2021 .250413$ 


\section{Revista Tópicos Educacionais}

Como, además, en el documento base "Propuesta de estructura curricular para la elaboración de las enseñanzas mínimas"(11) se anuncia que los criterios de evaluación no se fijarán para cada asignatura de cada curso, sino en $4^{\circ}$ y $6^{\circ}$ curso de Primaria y en $2^{\circ}$ y $4^{\circ}$ curso de ESO, en los libros de texto de unas editoriales se explicarán unos contenidos en unos cursos y en los de otras editoriales en otros, con lo que cambiar de colegio implicará el riesgo de repetir unos temas y de no ver otros. Peor, imposible.

8) El octavo gran error de la LOMLOE es establecer un único Título de Graduado en Educación Secundaria Obligatoria (GESO). Un único título sin ningún matiz, no permite diferenciar entre los alumnos que lo han obtenido de forma regular, es decir aprobando todos los cursos de la ESO, de los que lo han conseguido cursando una Formación Profesional Básica (FPB) o cursando un Programa de Diversificación Curricular (PDC), que son vías mucho más fáciles. Esto abre la puerta a que los alumnos no se esfuercen e intenten obtener el mismo título de la ESO por una vía más fácil.

9) El noveno error de la ley Celaá es complicar y burocratizar la tarea docente con numerosos nuevos términos e infinidad de competencias básicas a adquirir para cada uno de los objetivos educativos. Los dos documentos base sobre los currículos, uno sobre la propuesta curricular(11) y otro sobre el perfil de salida al acabar cada etapa(12) han estado elaborados por teóricos de la psicología evolutiva de la educación y, en lugar de especificar lo que los alumnos han de aprender en cada etapa, han introducido decenas de nuevos términos que ya han creado inseguridad a los profesionales de la educación, sobre lo que habrán de hacer. Ejemplos de estos nuevos términos son: "Perfil de Salida de la Educación Primaria (PSEP)", "Perfil de Salida de la Educación Básica (PSEB)", "descriptores operativos de las competencias clave", "competencia cognitiva o conocimientos", "competencia instrumental o destrezas", la "competencia actitudinal o actitudes", los tipos de competencias según las materias: "competencia en comunicación lingüística", "competencia plurilingüe", "competencia matemática y competencia en ciencia y tecnología (STEM)", "competencia digital", "competencia personal, social y de aprender a aprender", "competencia ciudadana", "competencia emprendedora" y la "competencia en conciencia y expresión culturales", etc. Luego vendrán las listas de centenares de formas concretas sobre cómo conseguir que los alumnos alcancen esas competencias, sin querer aceptar que en el momento de enseñar al docente le es imposible tener presente decenas y decenas de objetivos. 
En la presentación pública de esos dos documentos básicos, el viernes 26 de marzo de 2021, la ministra Isabel Celaá y su equipo (Cesar Coll, que ya fue colaborador en la LOGSE, y Elena Martín, ambos catedráticos de Psicología Evolutiva de la Educación) intentaron justificar su proyecto diciendo que hay que acabar con la enseñanza memorística, lo cual es sencillamente una falacia, porque ya nadie enseña a base de memorizar conceptos sin entenderlos y porque la memoria es imprescindible. Por ejemplo hay que aprender de memoria el nombre de las cosas, de los lugares, de los sucesos, de las personas relevantes, los vocabularios de las lenguas extranjeras, las normas gramaticales, etc. Por otro lado, sin memoria olvidaríamos lo aprendido, por lo que constantemente tendríamos que volver a aprender las cosas. Conviene recordar que es durante la adolescencia cuando los individuos tienen más memoria y que su desarrollo, como la de otras capacidades, se consigue con la práctica.

También defendieron la docencia compartida o codocencia, es decir que haya dos o más profesores en el aula y la enseñanza por ámbitos (Artículo 24.7 LOMLOE)(5) es decir impartir conjuntamente varias materias. Por ejemplo el ámbito científico-tecnológico comprende matemáticas, biología, geología, física, química y tecnología. Esto comporta que el alumnado no disponga de un especialista de cada una de las materias, que ha sido un gran logro en la enseñanza secundaria y que hasta ahora nadie había discutido. No existe ningún estudio que demuestre que la codocencia y la enseñanza por ámbitos sea mejor que el que haya un solo profesor en el aula especialista en la materia que enseña. Los legisladores no deberían pronunciarse sobre cómo se ha de enseñar, sino limitarse a decir lo que se ha de enseñar y luego evaluar si se ha conseguido, justamente las dos cosas que no se hacen en los dos documentos bases presentados. Lo más lamentable es que en el documento sobre la propuesta sobre el desarrollo curicular(11) se establece la promoción de curso entre $1^{\mathrm{o}}$ y $2^{\mathrm{o}}$ de ESO, uno de los grandes errores de la LOGSE que luego fue corregido. Según el calendario de aplicación de la LOMLOE(6) los nuevos currículos se presentarán el mes de septiembre de 2021, luego las editoriales publicarán los libros de texto y finalmente se empezarán a aplicar en el curso 2022/2023.

10) El décimo error de la LOMLOE es intentar acabar con la enseñanza concertada, con la enseñanza especial y con la enseñanza diferenciada. 
En el Artículo 109.2 de la LOMCE se establecía que las Administraciones educativas programarán la oferta educativa tomando en consideración la oferta existente en centros públicos y privados concertados y la “demanda social”. En cambio en la LOMLOE, en ese mismo Artículo, ha desaparecido el concepto de "demanda social", por lo que, aunque una familia quiera inscribir a un hijo en un determinado centro concertado en el que hay plazas libres, si la Administración no quiere que vaya a dicho centro, lo podrá hacer siempre que le ofrezca una plaza en otro centro, por ejemplo en un centro público. Por otro lado, en el Artículo 109 de la LOMLOE se ha añadido el punto 5 que dice: "Las Administraciones educativas promoverán un incremento progresivo de puestos escolares en la red de centros de titularidad pública.”.

Mientras que en el Artículo 4.3 de la LOE, que no fue modificado por la LOMCE, se establece que "se adoptará la atención a la diversidad como principio fundamental", en ese mismo artículo en la LOMLOE se establece, en cambio, que "se adoptará la educación inclusiva como principio fundamental, con el fin de atender a la diversidad de las necesidades de todo el alumnado, tanto del que tiene especiales dificultades de aprendizaje como del que tiene mayor capacidad y motivación para aprender.”. Esto implica la futura reducción de plazas para el alumnado con necesidad específica de apoyo educativo en los centros de educación especial, que mayoritariamente son centros concertados.

En el Artículo 84.3 de la LOMCE se establecía que los centros con enseñanza diferenciadas por sexos tenían derecho a pedir un concierto con las Administraciones educativas, mientras que en ese mismo artículo de la LOMOE no se permite concertar centros de enseñanza diferenciada.

Estas reducciones de la libertad de las familias para elegir el tipo de educación que quieren para sus hijos entre todos los centros educativos existentes, ya ha sido denunciada por la plataforma "Más plurales"(7) de las escuelas concertadas ante la Comisión Europea por atentar contra el Tratado de Funcionamiento de la Unión Europea y el artículo 27 de la Constitución Española al convertir la enseñanza concertada en subsidiaria de la pública.

\section{La LOMLOE una ley para conseguir una confederación de repúblicas}

Revista Tópicos Educacionais, Pernambuco, v. 27, n.01, p. 220-242, 2021. ISSN: 2448-0215.

https://periodicos.ufpe.br/revistas/topicoseducacionais/index DOI: 10.51359/2448-0215.2021.250413 


\section{Revista Tópicos Educacionais}

Todos los cambios establecidos por la ley de la ministra Celaá son entendibles si se acepta que su principal objetivo no es mejorar la enseñanza, sino conseguir que los alumnos conozcan y asuman los planteamientos ideológicos del Gobierno sobre cómo ha de ser la sociedad, qué valores éticos son los correctos y por qué el mejor modelo para España es el de convertirse en una confederación de repúblicas independientes, es decir un cambio profundo de la sociedad española en cuanto a su escala de valores y en cuanto a su pertenencia a un país, un cambio tan profundo que solo es posible mediante el adoctrinamiento escolar. Este objetivo es el que explica que los diez errores pedagógicos antes citados, no lo sean desde el objetivo político de conseguir un cambio de modelo de estado para España.

La LOMLOE ha establecido que el español o castellano ya no sea lengua vehicular de la enseñanza en toda España porque, para conseguir que España dé lugar a una serie de repúblicas independientes, es conveniente que los alumnos dejen de utilizar una lengua común, el español o castellano, y que solo utilicen la lengua regional cooficial, sea el catalán, el gallego o el vasco. El proceso está ya muy avanzado, por ejemplo en Cataluña está prohibido utilizar el español en la enseñanza desde 1983, ni en las Baleares ni en la Comunidad Valenciana es ya posible escolarizar a un niño en español ya que solo se utiliza el catalán, en el País Vasco y en Navarra está pasando lo mismo, es decir se está imponiendo el vasco y excluyendo el español como lengua vehicular de la enseñanza, y en Galicia se está disminuyendo las horas de clase que se imparten en español en favor del gallego.

La LOMLOE ha suprimido, antes de poderse estrenar, las pruebas externas al final de la ESO con valor académico, es decir con la necesidad de ser superadas para poder conseguir el título de la ESO, pruebas que dependerían directamente del Ministerio de Educación y que serían las mismas en toda España. Se han suprimido porque permitirían saber cómo van todos los alumnos de todas las Comunidades Autónomas en cada una de las materias, incluido el nivel de español, la visión positiva o negativa de la historia de España que han recibido y si han sido adoctrinados con ideologías políticas incompatibles con la Constitución Española, como es el deseo de secesión.

La LOMLOE quiere acabar con la enseñanza concertada porque los centros concertados tienen un ideario propio, muchos de ellos son católicos y, por lo tanto, pueden poner Revista Tópicos Educacionais, Pernambuco, v. 27, n.01, p. 220-242, 2021. ISSN: 2448-0215. https://periodicos.ufpe.br/revistas/topicoseducacionais/index DOI: $10.51359 / 2448-0215.2021 .250413$ 


\section{Revista Tópicos Educacionais}

resistencia a que muchas de las ideas del Gobierno sobre cuestiones morales lleguen al alumnado. También esa es la explicación de que no establezca una asignatura sobre las principales religiones con la misma categoría que las demás asignaturas. Por otro lado quiere acabar con la educación especial porque se trata de una enseñanza cara y porque, además, el 59\% de los centros de educación especial son concertados.

La preferencia por una confederación de repúblicas respecto a mantener la unidad territorial de España como una monarquía parlamentaria, podría deberse a que electoralmente podría ser rentable. Tras el fallecimiento de Franco en 1975, en España se optó por establecer 17 Comunidades Autónomas, cada una con su presidente, sus consejeros, su parlamento, sus elecciones, etc. Poco a poco se fueron transfiriendo las competencias en educación, en sanidad, en orden público, etc., sin que el Gobierno central se reservase su control. Las Comunidades que tenían una lengua regional propia optaron por exigirla en todos los sectores, tanto en las entidades públicas como en las actividades privadas, lo cual favoreció a los autóctonos y a sus empresas al eliminar la competencia. Por otro lado, a cambio de su apoyo al partido que quería gobernar en España, fuera de izquierdas (PSOE) o de derechas (PP), las comunidades con gobiernos secesionistas fueron consiguiendo privilegios. Actualmente en esas Comunidades Autónomas una gran parte de sus habitantes, sobre todo jóvenes, quieren ser un país independiente y otras Comunidades Autónomas están copiando ese modelo para igualmente favorecer a sus autóctonos. Debido a ello a los grandes partidos puede resultarles electoralmente rentable ofrecer un modelo basado en una confederación de repúblicas. Es un planteamiento irreal porque los partidos secesionistas (ERC, JxCat, PNV, Bildu, etc.) lo que pretenden es la independencia absoluta, no el formar parte de una federación española de repúblicas.

El perjuicio a los ciudadanos a este cambio es que este les comportaría pasar de vivir en un país grande (el cuarto de Europa), con mucho peso en las decisiones de la Unión Europea, con una gran diversidad de lenguas, de culturas y de planteamientos políticos e ideológicos, a vivir en un país muy pequeño, sin peso en la Unión Europea, de lengua única, de pensamiento único nacionalista, y en el que al ser pequeño, la pertenencia a determinadas familias y círculos sociales pesaría más que las capacidades. Los únicos que saldrían claramente beneficiados serían los políticos que promovieron este cambio, que pasaría a ser jefes de estado. 


\section{Cómo evitar los perjuicios de la LOMLOE}

Lo primero que se ha de tener en cuenta es que todo lo que se dice en la ley Celaá sobre lo que se debe hacer, puede ser incumplido por los gobiernos autonómicos sin que el Ministerio pueda hacer nada para evitarlo. Esto se debe a que la ministra Celaà, en su afán de complacer a los gobiernos autonómicos secesionistas, no ha establecido ningún mecanismo mediante el cual el Ministerio de Educación pueda intervenir y evitar un incumplimiento de la LOMLOE. Así pues, las Comunidades Autónomas constitucionalistas pueden establecer las medidas que consideren más adecuadas para mejorar la enseñanza. Las principales serían:

1. Establecer evaluaciones externas autonómicas al final de la Primaria y al final de la ESO, cuyo resultado debiera ser tenido en cuenta, en un determinado porcentaje, por los profesores en las calificaciones finales del alumno. Estas pruebas promoverían la cultura del esfuerzo, mejorarían el nivel académico de los alumnos, permitirían asegurar a las familias que las calificaciones que han recibido sus hijos por parte de los centros, realmente reflejan los niveles académicos alcanzados por ellos y acabarían con el "síndrome del alumno cliente" antes mencionado.

2. Hacer público los resultados de todos los centros en las evaluaciones autonómicas, en una escala de tres o cuatro niveles. Esta información permitiría orientar a las familias antes de la matriculación de sus hijos. Los ciudadanos tienen derecho a que la Administración les facilite esta información.

3. Permitir que las familias puedan elegir el centro escolar que deseen entre todos los centros públicos y concertados existentes en la Comunidad Autónoma. Esto, también denominado "distrito único", además de aumentar la libertad de elección de las familias, sería un estímulo para que todos los centros mejoraran los servicios educativos que ofrecen.

4. Permitir que las familias con hijos con necesidades educativas especiales puedan elegir el tipo de centro que prefieran. De esta manera las familias podrían decidir entre llevar a sus hijos a un centro ordinario, o a un centro de educación especial o bien a una educación combinada entre ambos.

Revista Tópicos Educacionais, Pernambuco, v. 27, n.01, p. 220-242, 2021. ISSN: 2448-0215.

https://periodicos.ufpe.br/revistas/topicoseducacionais/index DOI: $10.51359 / 2448-0215.2021 .250413$ 
5. Establecer que a partir de $3^{\circ}$ de ESO, es decir después de las pruebas de diagnóstico establecidas en la LOMLOE, los centros ofrezcan dos líneas de diferente dificultad. Esto permitiría poder atender mejor al alumnado, ya que se haría en función de sus capacidades y posteriores intereses.

6. Ponerse de acuerdo con otras Comunidades Autónomas para realizar la misma Prueba de Acceso a la Universidad. Esto permitiría mantener los mismos niveles de exigencia en todas ellas, lo cual es lo más lógico, dado que la calificación final del alumno en las PAU les permite acceder a cualquier Universidad de España.

7. Prohibir explícitamente la transmisión al alumnado de ideas políticas que no son compatibles con lo que establece la Constitución Española. Los centros escolares se han de mantener neutrales ante la confrontación que se establece entre los diferentes partidos políticos.

8. Establecer el derecho de los padres a ser consultados antes de que sus hijos reciban información sobre temas opinables. Los centros educativos han de ser neutrales respecto a los objetivos de los diferentes partidos políticos, las distintas creencias religiosas, las diferentes convicciones ideológicas, etc.

9. Mantener la continuidad de los conciertos a los centros con educación diferenciada. Aunque la LOMLOE lo prohíbe, el Tribunal Constitucional to ha considerado conforme a la Constitución Española. Por otro lado existen en infinidad de países, tanto en la enseñanza pública como en la concertada.

En las Comunidades Autónomas con gobiernos no constitucionalistas, los ciudadanos y las asociaciones de ciudadanos deberían seguir denunciando ante los tribunales de justicia a aquellos centros que no permitan el uso del español como lengua vehicular en la enseñanza en base al artículo 3 de la Constitución Española y de las sentencias de los tribunales, a los centros en los que se enseñen ideas incompatibles con la Constitución Española y a los centros en los que se adoctrine a los alumnos en ideas que son opinables. 


\section{Bibliografía}

(1) LOCE. LEY ORGÁNICA 10/2002, de 23 de diciembre, de Calidad de la Educación. (BOE del 24/12/2002). Ministerio de Educación, Cultura y Deporte. (PDF)

(2) LOE. LEY ORGANICA 2/2006, de 3 de mayo, de Edu $\neg$ cación. (BOE del 04/05/2006). Ministerio de Educación y Ciencia. (PDF)

(3) LOGSE. Ley 1/1990 de 3 de Octubre (BOE. de 4/10/1.990). Ley Orgánica de Ordenación General del Sistema Educativo. Ministerio de Educación. (PDF)

(4) LOMCE. Ley Orgánica 8/2013, de 9 de diciembre, para la mejora de la calidad educativa. (BOE del 10/12/2013). Ministerio de Educación, Cultura y Deporte. (PDF)

(5) LOMLOE. Ley Orgánica 3/2020, de 29 de diciembre, por la que se modifica la Ley Orgánica 2/2006, de 3 de mayo, de Educación. (BOE del 30/12/2020). Ministerio de Educación y Formación Profesional. (PDF)

(6) LOMLOE. Calendario de implantación (PDF)

(7) Más plurales. Plataforma de las escuelas concertadas. (WEB)

(8) PISA. 2015. Informe PISA de la OCDE sobre Portugal (PDF)

(9) PISA 2018. Programa para la Evaluación Internacional de los Estudiantes. Informe español (WEB)

(10) PISA 2018. Results in the world. (WEB)

(11) Propuesta de estructura curricular para la elaboración de las enseñanzas mínimas. 2021. Secretaría de Estado de Educación. Ministerio de Educación y Formación Profesional. (PDF)

(12) Perfil de salida del alumnado al término de la educación básica. 2021. Secretaría de Estado de Educación. Ministerio de Educación y Formación Profesional. (PDF)

(13) Sánchez Lissen, E. 2020. La influencia de las evaluaciones internacionales sobre las políticas educativas: una mirada a la educación en Europa desde PISA. Laplage em Revista (Sorocaba), vol.6, n.2: 19-36 (PDF) 
Revista Tópicos Educacionais

Revista do Programa de Pós-Graduação em Educação

da Universidade Federal de Pernambuco (UFPE)

Recebido em maio de 2021.

Aprovado em junho de 2021.

Revista Tópicos Educacionais, Pernambuco, v. 27, n.01, p. 220-242, 2021. ISSN: 2448-0215.

https://periodicos.ufpe.br/revistas/topicoseducacionais/index 